Inferring rubella outbreak risk from seroprevalence data in Belgium Peer-reviewed author version

ABRAMS, Steven; Kourkouni, Eleni; Sabbe, Martine; Beutels, Philippe \& HENS, Niel (2016) Inferring rubella outbreak risk from seroprevalence data in Belgium. In:

VACCINE, 34 (50), pag. 6187-6192.

DOI: $10.1016 / j$. vaccine.2016.10.072

Handle: http://hdl.handle.net/1942/22602 


\section{Inferring Rubella Outbreak Risk From Seroprevalence Data in Belgium}

2

\section{Steven Abrams*}

4

Diepenbeek, Belgium.

\section{Eleni Kourkouni}

\section{Martine Sabbe}

\section{Philippe Beutels}

\section{Niel Hens}

Interuniversity Institute for Biostatistics and statistical Bioinformatics, UHasselt (Hasselt University),

Center for Clinical Epidemiology and Outcomes Research (CLEO), Athens, Greece.

Service of Epidemiology of Infectious Diseases, Department of Public Health and Surveillance, Scientific Institute for Public Health, Brussels, Belgium.

Centre for Health Economics Research and Modeling Infectious Diseases and Centre for the Evaluation of Vaccination, Vaccine \& Infectious Disease Institute, University of Antwerp, Wilrijk, Belgium, and School of Public Health and Community Medicine, The University of New South Wales, Sydney, Australia.

Interuniversity Institute for Biostatistics and statistical Bioinformatics, UHasselt (Hasselt University), Diepenbeek, Belgium, Centre for Health Economics Research and Modeling Infectious Diseases and Centre for the Evaluation of Vaccination, Vaccine \& Infectious Disease Institute, University of Antwerp, Wilrijk, Belgium, and Department of Epidemiology and Social Medicine, University of Antwerp, Wilrijk, Belgium.

\footnotetext{
*Corresponding author at: Interuniversity Institute for Biostatistics and statistical Bioinformatics, UHasselt (Hasselt University), Agoralaan Building D, BE3590 Diepenbeek, Belgium. Tel: +3211268235

E-mail address:steven.abrams@uhasselt.be
} 
Abstract. Rubella is usually a mild disease for which infections often pass by unnoticed. In approximately $50 \%$ of the cases, there are no or only few clinical symptoms. However, rubella contracted during early pregnancy could lead to spontaneous abortion, to central nervous system defects, or to one of a range of other serious and debilitating conditions in a newborn such as the congenital rubella syndrome. Before the introduction of mass vaccination, rubella was a common childhood infection occurring all over the world. However, since the introduction of rubella antigen-containing vaccines, the incidence of rubella has declined dramatically in high-income countries. Recent large-scale mumps outbreaks, one of the components in the combined measles-mumps-rubella vaccine, occurring in countries throughout Europe with high vaccination coverage, provide evidence of pathogen-specific waning of vaccineinduced immunity and primary vaccine failure. In addition, recent measles outbreaks affecting populations with suboptimal vaccination coverages stress the importance of maintaining high vaccination coverages. In this paper, we focus on the assessment of rubella outbreak risk using a previously developed method to identify geographic regions of high outbreak potential. The methodology relies on 2006 rubella seroprevalence data and vaccination coverage data from Belgium and information on primary and secondary vaccine failure obtained from extensive literature reviews. We estimated the rubella outbreak risk in Belgium to be low, however maintaining high levels of immunisation and surveillance are of utmost importance to avoid future outbreaks.

Keywords: disease outbreaks; effective reproduction number; next-generation operator; serology; social contact hypothesis; vaccines

\section{Introduction}

Rubella infection causes usually mild or asymptomatic disease. However, rubella vaccination is of major public health interest because rubella contracted during early to mid-pregnancy could lead to spontaneous abortion, central nervous system defects, or one of a range of other serious and debilitating conditions in a newborn (e.g., congenital rubella syndrome (CRS) leading to heart, brain, ear and eye problems). The rubella vaccine has been used successfully in vaccination programs around the world targeted at girls only or girls and boys combined, and usually successfully accompanied by catch-up vaccination up to pre-adolescence [1]. Rubella vaccination has been available in Belgium since 1974 for teenage girls only until 1985 when universal 
1

vaccination with the combined measles-mumps-rubella (MMR) vaccine was recommended and made available free of charge on the routine program at age 15 months only. In 1995, the second dose of MMR was added to the program at age 11-12 years [2]. Since 2003 the age at first dose administration was lowered to 12-13 months, in an attempt to offer protection as early as possible after waning of residual maternal antibodies.

Trivalent measles, mumps and rubella immunisation has been widely implemented throughout Europe and the incidence of these diseases has declined dramatically. However, local and large-scale outbreaks of measles have been reported in recent years and have been attributed to suboptimal vaccination coverage [see, e.g., 35]. In addition to measles outbreaks, mumps outbreaks have been reported in highly vaccinated populations during recent years [see, e.g., 6,7], raising concerns about mumps vaccine failure. Whereas there are no recent reports on rubella outbreaks in Belgium, in Japan, an outbreak of 15,000 cases of rubella and 43 cases of CRS was observed. This rubella resurgence mainly affected unvaccinated adult men and women belonging to cohorts in which vaccination coverages were relatively low [8]. In 2012, rubella outbreaks were also reported in Poland and Romania $[9,10]$. On the other hand, Finland was documented to be the first country in which rubella is eliminated as a result of a 12 year, 2-dose MMR vaccination program [11].

Despite MMR vaccination efforts throughout the world, it remains of interest to determine whether measles, mumps and rubella resurgence is possible in countries with high vaccination coverage. Recently, Abrams et al. [12] and Hens et al. [13] have conducted such studies for mumps and measles in Belgium. In the absence of recent serological data, they showed that a multi-cohort model can be used, combining previously collected serological survey data and vaccination coverage information, as well as data on social contact behavior. These data are then combined with biomedical information on the duration of maternal immunity and of primary and secondary vaccine failure, both based on extensive literature reviews. The objective of the current paper is to complement the aforementioned analyses with an outbreak risk assessment for rubella in Belgium. More specifically, our aim is to identify Belgian regions of high outbreak potential based on regional estimates of the effective reproduction number $R$ thereby closely following the approach by Abrams et al. [12]. 
1

2

3

4

5

6

\section{Material and methods}

\subsection{Data sources}

\subsubsection{Serologicalsurvey data}

A previously published Belgian serological data set on rubella from 2006 serves as the main data sourcefor our analyses. This database was compiled using a multi-tiered approach to collect a sufficient number of geographically and age and gender representative serum samples $(n=3823)$ [14]. Samples were tested for the presence of immunoglobulin $\mathrm{G}$ antibodies against rubella using a commercial ELISA (Enzygnost, Siemens, Germany). Hence, these serological data contain information about past rubella immunisation, either by vaccination (passive immunisation) or natural infection (active immunisation). Equivocal and missing test results were excluded from our statistical analyses.

\subsubsection{Vaccination coverage information}

After the first year of life, Belgian infants gain vaccine-induced immunity through vaccination at the age of about 12 months (first dose) and about 12 years (second dose; 10 years-13 years). In order to quantify vaccineinduced immunity we rely on vaccination coverage estimates for both recommended MMR doses in this 2-dose MMR program (see Table A1 in Appendix A).

\subsubsection{Social contact data}

We integrated social contact data from Belgium collected in the European POLYMOD study $[15,16]$ in the estimation of the age-specific relative incidence of rubella resurgence. Age-dependent contact rates are estimated from Belgian empirical data according to a bivariate smoothing approach [17]. These social contact rates provide information on the mixing behaviour of individuals between different age-groups important to explain the spread of airborne infections such as rubella in the target population.

\subsubsection{Primary and secondary vaccine failure}

Extensive literature searches in PubMed and ISI Web of Knowledge and meta-analyses were conducted to obtain estimates for seroconversion (as a proxy for primary vaccine failure) and waning rubella immunity following MMR vaccination (see Appendix A for details on inclusion criteria and estimates and meta-analysis in 
1

Appendix B). The seroconversion rate is assumed identical after first and second MMR dose, and estimated to be $0.984(95 \% \mathrm{Cl}:[0.974,0.999])$ in line with estimates obtained by Wood et al. [18] relying on serial serological data. Note that seropositivity for anti-rubella IgG was used as a proxy for natural infection or vaccine-induced protection, and seronegativity as a proxy for susceptibility. A common annual waning rate $\hat{\gamma}=$ $0.016[0.014,0.018]$ is obtained from the literature review. Alternatively, we estimated an overall waning rate for rubella based on Belgian serological data anno 2006 in the age interval 13 to 20 years in order to select individuals vaccinated twice with the combined MMR vaccine (i.e., under the assumption that rubella circulation was interrupted after the introduction of mass vaccination). More specifically, the log-tra ns formed antibody levels for seropositive individuals were linearly modelled as a function of age, yielding an exponentia I annual waning rate of $\hat{\gamma}=0.003[0.000,0.026]$. The estimated waning rate derived from the second approach is very close to the estimate obtained by Wood et al. [18] (i.e., $0.002[0.000,0.004]$ ), albeit less precise, and will be used in our analyses as a conservative approach.

Although evidence for waning of vaccine-induced immunity exists, rubella infections are generally accepted to induce lifelong immunity [19], which we assume here, yielding a conservative approach when estimating rubella outbreak risk.

\subsection{Multi-cohort model formulation}

We use a multi-cohort model for the assessment of rubella outbreak risk in Belgium. We closely follow the work by Abrams et al. [13] and Hens et al. [13]. Briefly, with $\pi(a, t)$ representing the proportion seropositive individuals of age $a$ at calendar time $t$ in the population, suppressing dependence on other individual covariate information for the time being, the multi-cohort model is partly based on the following set of equations for $\pi(a, t)=1-S(a, t)$, with $S(a, t)$ the age- and time-dependent susceptible fraction:

$$
\pi(a, t)=\left\{\begin{array}{lr}
\exp (-\eta a) \times[1-S(0, t-a)] & \text { if } 0 \leq a<1, \\
\exp \left[-\gamma_{1}(a-1)\right] \times \rho v_{1}(t-a+1) & \text { if } 1 \leq a<12, \\
\exp \left[-\gamma_{2}(a-12)\right] \times \rho v_{2}(t-a+12) & \text { if } 12 \leq a
\end{array}\right.
$$

where $\eta$ represents the rate at which maternal antibodies decay, $\rho$ the seroconversion rate, $\gamma_{1}$ and $\gamma_{2}$ waning rates of vaccine-induced immunity after the first (MMR1) and second dose of the MMR vaccine $(\mathrm{MMR} 2), v_{1}($.$) ,$ $v_{2}($.$) the time-dependent proportion vaccinated with MMR1 and MMR2, and S(0, t-a)$ is the proportion of susceptible newborns at calendar time $(t-a)$. In order to calculate the age-dependent proportion of 
1

2

susceptible individuals at calendar time $t=y r^{R}$, we need to apply and adapt the aforementioned cohort model as follows. First, for individuals aged $\left[13+\left(y r^{R}-2006\right)\right]$ or older in reference year $y r^{R}$ (i.e., those aged 13 years or older in 2006) we account for vaccine-induced and naturally acquired immunity by combining time-specific vaccination coverage information with $S(a, t)$ derived from the 2006 serological data. In or der to propagate $S(a, t)$ to future years, waning of vaccine-induced immunity is accommodated through multiplication with a factor $\exp \left[-\gamma_{2}\left(a-a_{0}\right)\right]$, i.e., $a_{0}$ the individual's age in 2006 , and natural immunity is assumed to persist for life. Second, for individuals younger than $\left[13+\left(y r^{R}-2006\right)\right]$ years of age, we cannot use the 2006 serology to estimate the proportion susceptible, because a second MMR dose has been offered to these children later on. Therefore, we need to rely entirely on the more recent vaccination coverage data. In order to do so, we use the equations in (1) to determine the age-dependent proportion susceptible (see also Appendix C). Note that we also adapted the third equation to account for those who received the first dose only. The equations in (1) are based on the assumption that circulation is interrupted since 1993 (i.e., serological data for individuals aged 13 or more in 2006 are included in the estimation of the susceptibility profile, and hence transmission resulting in humoral immunity in these age groups is accounted for). Transmission is not accounted for in the estimation of susceptibility in those aged 12 or less in 2006. Considering the absence of reported rubella outbreaks in Belgium since 1994, this seems unlikely to have a large impact.

In order to estimate the seroprevalence, data from a previously described seroprevalence study conducted in 2006 is used containing binary current status data with respect to past rubella immunisation (see Section 2.1.1). A generalised additive model (GAM) [20,21] with complementary log-log link function, denoted by $\operatorname{clog} \log ($.$) , is considered to model the observed seroprevalence as a function of the individual's age a$, gender $g$ and spatial location $(x, y)$ :

$$
\operatorname{cog} \log [\pi(a, x, y, g)]=f(a, x, y, g),
$$

where $f($.$) is a smooth function and \pi(a, x, y, g)$ is the proportion of seropositives of age $a$ with spatial coordinates $(x, y)$ and gender $g$. The coordinates $(x, y)$ refer to the centroids of the municipalities in which test subjects reside, if available; otherwise spatial locations of the test laboratories are used as a proxy. Severa I submodels of equation (2) were considered and a model comparison was performed based on Akaike's Information Criterion [AIC, 22]. The smooth function $f($.$) was decomposed in smooth components s_{i}(),. i=1$, 
12 , which were fitted using one-dimensional cubic splines and two-dimensional thin-plate regression splines,

2 respectively, and/or components $t e(.,$.$) referring to tensor product thin-plate regression splines allowing for$

3 differential smoothing along the two dimensions. As a result, the GAM in (2) enables estimating the age-,

4 gender- and location-specific proportion of susceptible individuals in Belgium anno 2006, denoted by

$5 S(a, 2006, x, y, g)=1-\pi(a, x, y, g)$, by averaging data points with their neighbours. Consequently, susceptibility predictions for reference year $y r^{R}$ are obtained after multiplying the age-dependent fraction of seropositive individuals with a factor $\exp \left[-\gamma\left(y r^{R}-2006\right)_{+}\right]$which represents an exponential decay function with decay rate $\gamma$ and $(u)_{+}=u$ if and only if $u>0$. The a nalyses based on serology from 2006 are restricted to subjects aged at least 13 years in 2006 , since predictions for younger ages are likely influenced by samples for which the second vaccine dose was not yet administered at the time of data collection. Therefore, predictions of the proportion of susceptible individuals in reference year $y r^{R}$ based on the GAM are solely available for individuals aged $\left[13+\left(y r^{R}-2006\right)\right]$ years and above. Susceptibility in younger age groups is inferred from available Belgian vaccination coverage information (see Appendix C). Finally, the estimated spatial age- and time-dependent susceptibility profile in $y r^{R}$ is us ed to infer the effective reproduction number $R$ (see Appendix D).

\section{Results}

\subsection{Local effective reproduction numbers in Belgium}

Several GAMs are fitted to the Belgian rubella serology using the R package "mgcv" package in order to determine the age- and location-specific susceptibility profiles (Table 1 ). 
Table 1. Generalized additive models fitted to the Belgian seroprevalence data anno 2006 on rubella infection s with corresponding AIC-values; individual's age $a$, gender $g$ and spatial location $(x, y) ; s_{i}(),. i=1,2$, onedimensional cubic splines and two-dimensional thin-plate regression splines, respectively, $t e(.,$.$) tensor$ product thin-plate regression splines.

\begin{tabular}{|clc|}
\hline Model & Linear predictor & AlC \\
\hline$(1)$ & $t e(x, y, a, b y=g)+t e(x, y, a, b y=1-g)$ & 899.98 \\
$(2)$ & $t e(x, y, a)$ & 932.89 \\
$(3)$ & $s_{1}(a)+t e(x, y)$ & 935.04 \\
$(4)$ & $s_{1}(a)+s_{2}(x, y)$ & 933.15 \\
$(5)$ & $s_{1}(a)$ & 969.86 \\
\hline
\end{tabular}

6

5

(

Model (1) outperforms all other fitted models based on AIC. Hence, gender is an important variable in explaining the observed seroprevalence data. Henceforth, gender-specific susceptibility profiles for reference year $y r^{R}=2016$ are derived based on model (1) and relying on vaccination coverages, seroconversion and waning rates, and subsequently used in the assessment of rubella outbreak risk in Belgium. A parametric bootstrap approach is used to construct 1,000 bootstrap samples, and model (1) is fitted to each of the generated samples. Furthermore, for each bootstrap sample and municipality, vaccination coverages are randomly sampled from a normal distribution with $95 \%$ confidence intervals approximately equal to those reported in Table A1. In order to end up with a smooth susceptibility curve for each municipality, a gradual evolution of the vaccination coverages is assumed and an interpolating-spline model is fitted to each of the generated data sets. In Figure 1, the estimated susceptibility profiles in 2016 for males (left panel) and fema les (right panel) are shown for the five largest Belgian cities (i.e., Ghent, Antwerp, Brussels, Liège and Charleroi) for the purpose of illustration. The profiles are similarin shape; the susceptibility is higher in Charleroi and Brussels than in other cities. In the estimation of the spatial effective reproduction numbers $R$, a weighted average of gender and age-specific susceptibility is calculated per municipality with weights equal to the age-dependent proportion of males/females. 

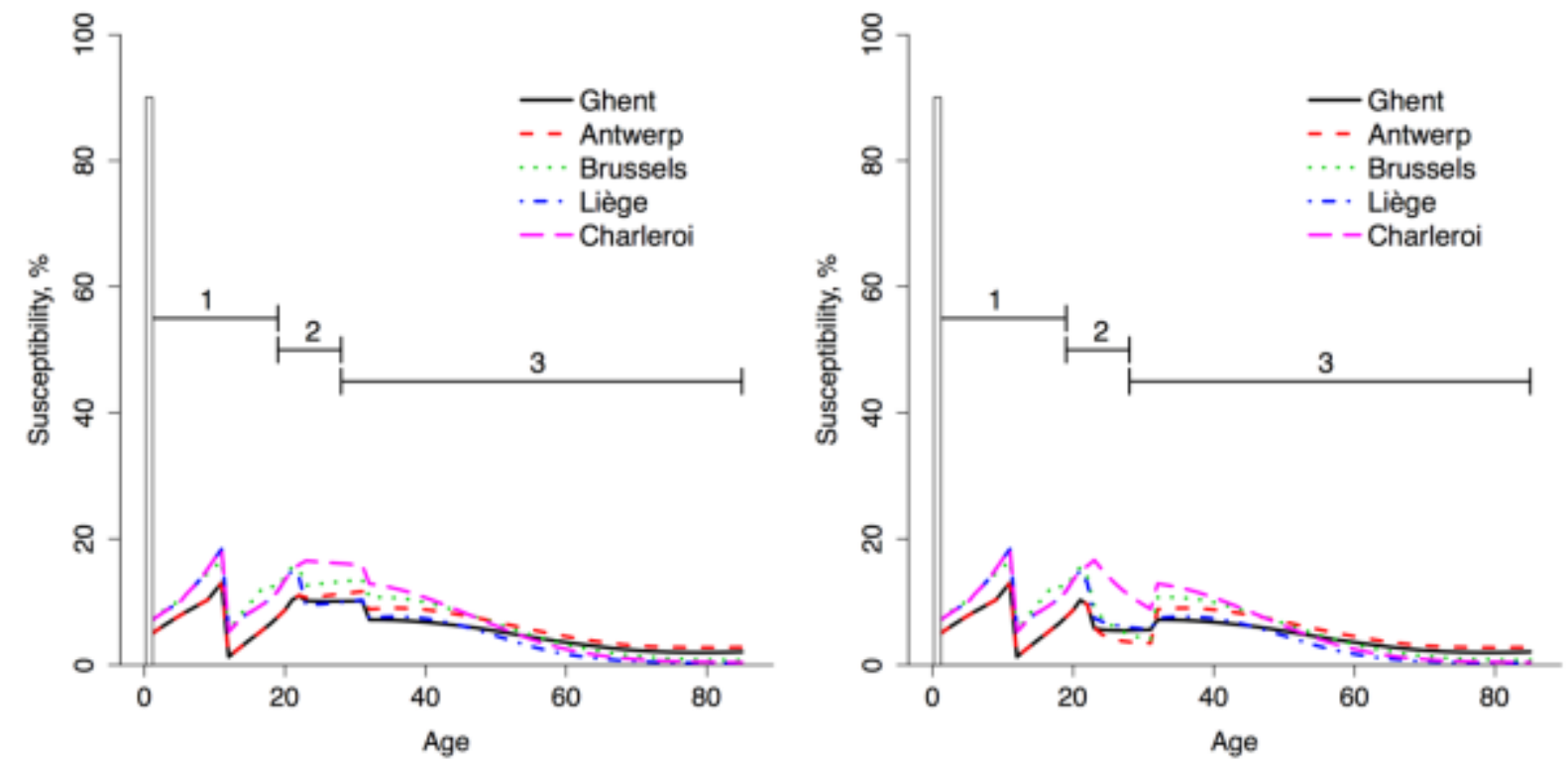

Figure 1. Estimated susceptibility to rubella infection in the five largest Belgian cities: Ghent, Antwerp, Brussels, Liège and Charleroi in 2016 for males (left panel) and females (right panel). The susceptibility curve is based on (1) coverage information + waning of vaccine-induced immunity; (2) serology with waning of vaccine-induced immunity; and (3) serology with lifelong natural immunity.

7 Estimates for the basic reproduction $R_{0}$ in the literature range from 4 to $8[23,24]$. Based on the left panel of

8 Figure 2, displaying the estimated effective reproduction numbers for the intermediate setting with $R_{0}=6$,

9 one can conclude that rubella outbreak riskis relatively low in all Belgian municipalities with slightly higher R values in Sivry-Rance (0.835), Chimay (0.843) and Momignies (0.916) (red regions). Results of a sensitivity analysis with values of $R_{0}$ ranging from 4 to 8 are presented in the right panel of Figure 2. Obviously, a higher value for $R_{0}$ implies higher values of $R$. Again, the rubella outbreak risk is estimated to be quite low, al beit that in the extreme case of $R_{0}=8$, the effective reproduction numbers tend to exceed the threshold value one in 10 municipalities, mainly in Brussels and the Walloon region where vaccination coverage is lower relative to Flanders. 


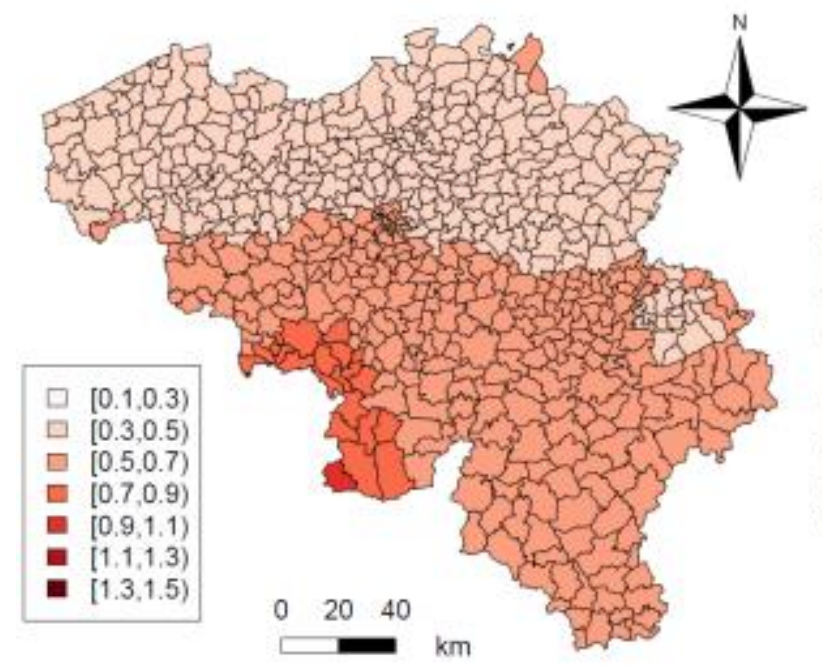

1

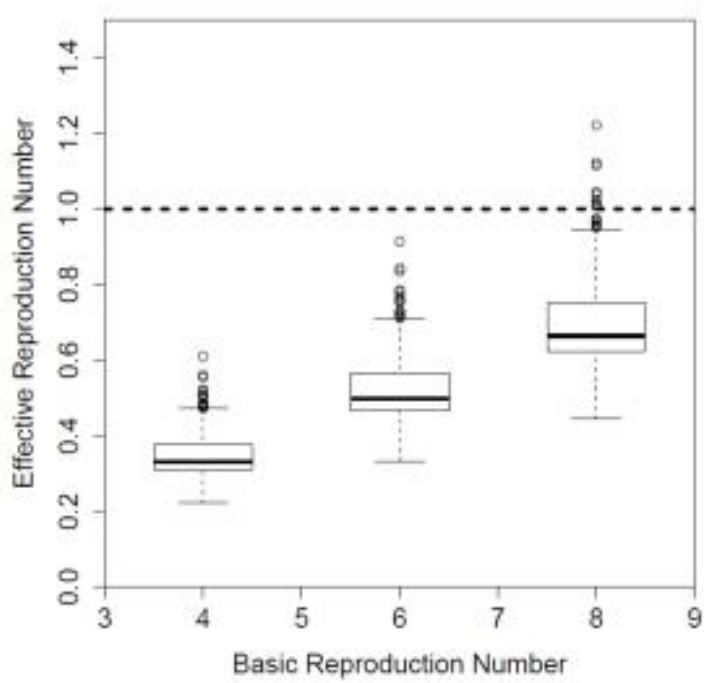

Figure 2. Estimated effective reproduction numbers for rubella in the Belgian municipalities on a spatial map for $R_{0}=6$ (left panel) and spatial boxplots of the estimated effective reproduction numbers with basic reproduction number Ro equal to 4,6 or 8 .

5

$6 \quad 3.2$. Relative incidence in a future rubella outbreak

7 The expected age-dependent relative incidence of a rubella outbreak upon importation of a rubella case is

8 calculated based on the susceptibility in the aforementioned five Belgian cities (Figure 3). Infants younger than

9 one year of age are the most vulnerable to rubella infection due to a rapid decay of maternal protection as

demonstrated by Leuridan et al. [25] and Goeyvaerts et al. [26]. The incidence in the age groups 15-19 and 20-

1124 years is expected to be highest and results from an increased susceptibility together with higher social contact frequencies in these age groups. 


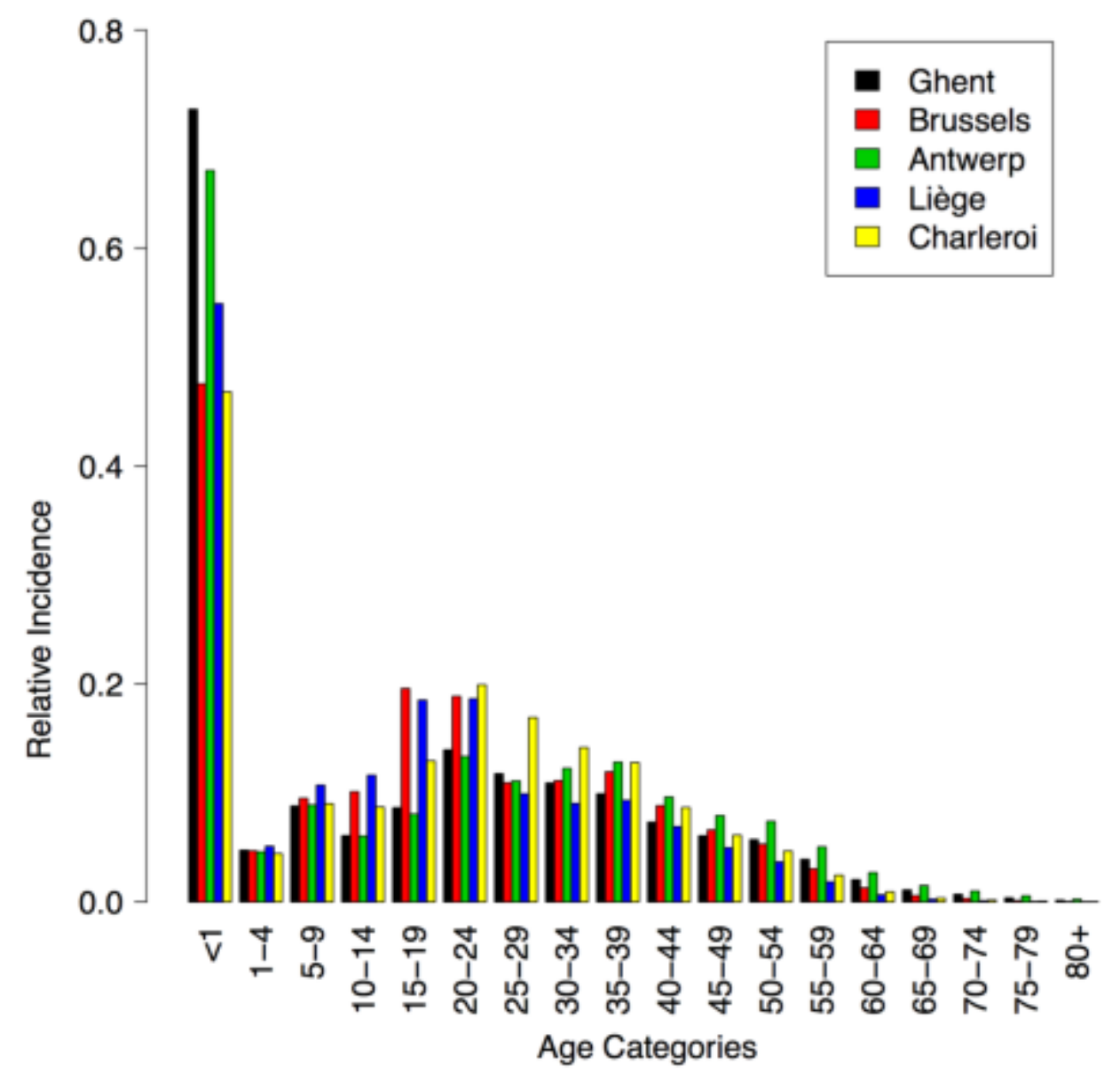

Figure 3. Expected age-specific relative incidence of a re-emergence of rubella in Belgium for five cities: Ghent, Antwerp, Brussels, Liège and Charleroi.

\section{Discussion}

In this paper, we investigated the possibility of rubella resurgence in the Belgian population. Differences in

8 estimated susceptibility between males and females reflect the sequence of female only (prior to 1985) and gender neutral (from 1985 onwards) rubella immunisation activities in Belgium. Based on the projected susceptibility in 2016, the effective reproduction numbers in Belgian municipalities are estimated relying on a range of previously published basic reproduction numbers. Al though the risk of large-scale rubella outbreaks is found to be low at the moment, monitoring rubella seropositivity in the population remains of crucial importance in order to evaluate the risk of outbreaks in the future. The low outbreak risk is mainly driven by pre-existing vaccine-induced immunity in females from cohorts vaccinated with a monovalent rubella vaccine prior to the administration of the combined MMR vaccine. However, seropositivity of vaccinated females in these cohorts is not subjected to waning of vaccine-induced immunity between 2006 and 2016 as reliable coverage estimates for the period 1973-1985 are unavailable. Therefore, seropositivity in females born before 
1

1985 is assumed to be the result of natural infection without waning rather than immunisation through vaccination. This could lead to an underestimation of susceptibility in the population. In addition, the seroconversion rate after MMR vaccination for rubella is estimated to be almost one (see Table A2). This implies that a large part of the vaccinated population is protected against rubella infection immediately after vaccination. Exponential waning rates are estimated to be quite small compared to those reported by Abrams et al. [12] for mumps (i.e., 0.043 and 0.021 after MMR1 and MMR2, respectively). As individuals with naturally acquired immunity to rubella start to disappear as they increasingly die, and waning of vaccine-induced immunity enlarges the susceptible proportion in the population, rubella outbreaks are not unlikely to occur in the future. This is especially important for health services, as rubella outbreaks can lead to an increased incidence of CRS in newborns [see, e.g., 27]. However, until now, rubella outbreaks in the vaccination era are mainly affecting countries with suboptimal vaccination levels.

In general, the methodology presented in this manuscript enables the estimation of outbreak risk for vaccinepreventable diseases in highly vaccinated populations. Our model is informed by data that is commonly available at the country level, namely (spatial) serological data, vaccination coverage information and social contact data, and accounts for loss of maternal immunity, primary vaccine failure and waning of vaccineinduced immunity. Nevertheless, our study suffers from several limitations. A proportion of persons who do not have detectable antibodies may have a level of protection via cellular immunity [28], but this proportion remains unknown and could therefore not be taken into account. However, if outbreaks would emerge we could at least partially estimate the role of cellular immunity as a potential dampening factor in the outbreak size. Moreover, ELISA results do not perfectly correlate with the plaque reduction neutralisation values, used in the few other existing studies relating circulating antibodies to clinical protection against rubella, which are generally more sensitive [see, e.g., 29]. Naturally acquired immunity after rubella infection is believed to be lifelong. However, any deviation from this assumption is easily incorporated, leaving a larger part of the population unprotected against new rubella infections. The latter situation increases the estimated reproduction numbers and cons equently enlarges the efforts required to prevent future rubella outbreaks.

Although the location- and age-dependent susceptibility profiles are constructed based upon various data sources and therefore apprehensive graphical indica tions of regions with high outbreak risk are provided, one 
1

should remain cautious about over-interpreting these spatial results. The spatial resolution of the various data sources we used was limited. The analysis of the serological data clearly indicated spatially heterogeneous serological profiles for the different infections, with differences between Flanders, Wallonia and Brussels mainly driven by differences in vaccination coverages, which are in line with those reported by others [see references in Table A1 and 30]. Coverage data in the Belgian regions was fragmented, leading to a substantial amount of uncertainty about the susceptibility in young age groups, and the spatial distribution of our social contact data was not detailed enough due to sample size restrictions, limiting spatial estimates of susceptibility. More detailed information at a smaller (spatial) resolution could improve prediction, a nd would enable us to account for spatial correlation in vaccine activities and correlation of vaccine activities ( $f$ irst a nd second dose) within individuals. Finally, susceptibility was only allowed to change over time as a result of waning of vaccine-induced immunity, and therefore, very rarely, decrea ses in susceptibility caused by spora dic infections over the years areignored.

Currently rubella surveillance in Belgium is suboptimal and relies mainly on sentinel surveillance of congeni ta I rubella. Periodic monitoring of seropositivity and inferring outbreak risk as conducted in this study remains desirable to complement clinical surveillance and detect effects such as waning of vaccine-induced immunity.

\section{Acknowledgements}

This work was supported by the Research Fund of Hasselt University (grant BOF11NI31 to SA). NH and PB acknowledge support from the Scientific Chair in Evidence-based Vaccinology, financed by a gift from Pfizer, and the Antwerp Study Centre for Infectious Diseases (ASCID), both at the University of Antwerp.

Conflicts of interest: none declared.

Authors' contribution to the manuscript:

SA, EK and NH performed the analyses and interpreted the results. SA and NH wrote the initial draft of the manuscript after which all authors critically revised the articleand contributed to drafting the final manuscript. All authors read and approved the final manuscript. 


\section{Supplementary materials}

Supplementary materials associated with this article can be found in Appendix A-F.

\section{References}

[1] Bana tvala JE, Brown DW. Rubella. La ncet 2004;363:1127-37.

[2] Beutels P, Van Damme P, Van Casteren V, Gay N, De Schrijver K, Meheus A. The difficult quest for data on "vanishing " vaccine-preventable infections in Europe: the ca se of measles in Flanders (Belgium). Vaccine 2002;20:3551-9.

[3] Antona D, Lévy-Bruhl D, Baudon C, Freymuth F, La my M, Ma ine C, Floret D, Pa rent du Chatelet I. Mea sles elimination efforts and 2008-2011 outbreak, France. Emerg Infect Diseases 2013;19:357-64.

[4] Baugh V, Figueroa J, Bosanquet J, Kemsley P, Addiman S, Turbitt D. Ongoing measles outbreak in Orth odox Jewish community, London, UK. Emerg Infect Diseases 2013;19:1707-9.

[5] Bra eye T, Sa bbe M, Hutse V, Flipse W, Godderis L, Top G. Obstacles in measles elimination: a n in -depth description of a measles outbreakin Ghent, Belgium, s pring 2011. Arch Public Health 2013;71:17.

[6] Cohen C, White JM, Sa vage EJ, Glynn JR, Choi Y, Andrews N, Brown D, Ramsay ME. Vaccine effectiveness estimates, 2004-2005 mumps outbreak, England. Emerg Infect Diseases 2007;13:12-7.

[7] Flipse W, De Schrijver K. Mumps outbreak among university students in 2012 [in Dutch]. Vlaams Infectiebulletin 2013;85:Article 2.

[8] Uji ie M, Na bae K, Shobayashi T. Rubella outbreak in Japan. Lancet 2014;383:1460-1.

[9] Pa ra dowska-Stankiewicz I, Czarkowski MP, Derrough T, Stefanoff P. Ongoing outbreak of rub ella a mo ng you ng male a dults in Poland: increased risk of congenital rubella infections. Euro Surveill 2013;18:pii:20485.

[10] Janta D, Stanescu A, Lupulescu E, Molnar G, Pistol A. Ongoing rubella outbreak a mong adolescents in Salaj, Ro ma nia, September 2011-January 2012. Euro Surveill 2012;17:pii=20089.

[11] Peltola H, Heinonen OP, Va lle M, Pa unio M, Virtanen M, Ka ra nko V, Cantell K. The elimination of indigenous measles, mumps, and rubella from Finland by a 12-year, two-dose va ccination program. N Engl J Med 1994;331:1397-402.

[12] Abra ms S, Beutels $P$, Hens N. As sessing mumps outbreak risk in highly va ccinated populations using spatial seroprevalence data. Am J Epidemiol 2014;179:1006-17.

[13] Hens N, Abrams S, Sa ntermans E, The eten H, Goeyvaerts N, Lernout T, Leuridan E, Van Kerckhove K, Goossens H, Van Da mme P, Beutels P. As sessing the risk of measles resurgence in a highly va ccinated population: Belgium ann o 2013. Euro Surveill 2015;20:pii=20998.

[14] Theeten $\mathrm{H}$, Hutse V, Hens N, Yavuz Y, Hoppenbrouwers PBK, VranckxR, Van Damme P. Are we hitting immunity ta rgets? The 2006 age-specific seroprevalence of measles, mumps, rubella, diph theria and tetan us in Belgium. Epidemiol Infect 2011;139:494-504.

[15] Mossong J, Hens N, Jit M, Beutels P, Auranen K, Mikolajczyk R, Massari M, Salmaso S, Scalia Tomba G, Wallinga J, Heijne J, Sa dkowska-Todys M, Rosinska M, Edmunds WJ. Social contacts a nd mixing patterns relevant to the spread of infectious diseases. PLoS Med 2008;5:381-91.

[16] Hens N, Aye le GM, Goeyva erts N, Aerts M, Mossong J, Edmunds JW, Be utels P. Es timating the impact of s chool closure on social mixing behaviour and the transmission of close contact infections in eights European countries. BMC infectious diseases 2009;9:187.

[17] Goeyva erts N, Hens N, Ogunjimi B, Aerts M, ShkedyZ, Van Damme P, Be utels P. Es timating infectious disease pa ra meters from data on social contacts and serological status. J R Stat Soc Series C 2010;59:255-77. 
[18] Wood JG, Goeyvaerts N, Maclntyre CR, Menzies RI, McIntyre PB, Hens N. Estimating va ccine coverage from s erial triva riate serologic data in the presence of waning immunity. Epidemiol 2015;26:381-9.

[19] Plotkin SA, Orenstein WA, and Offit PA. Vaccines. 5th ed. Philadelphia: Saunders; 2008.

[20] Has tie T, Tibshirani R. Generalized a dditive models. London: Chapman \& Ha Il; 1990.

[21] Wood SN. Generalized Additive Models: a n Introduction with R. Boca Raton: Chapman \& Hall/CRC; 2006.

[22] Akaike H. Information theory a nd an extension of the maximum likelihood principle. In B. N. Petrova nd F. Cs aki (Eds.), 2nd International Symposium on Information Theory, pp. 267-281; 1973.

[23] Anders on RM, May RM. Infectious Diseases of Humans; Dynamics a nd Control. Oxford: Oxford University Press; 1991.

[24] Fa rrington P. Modeling Epidemics. Milton Keynes: The Open University; 2003.

[25] Leuridan E, Hens N, Hutse V, Aerts M, Van Damme P. Kinetics of maternal a ntibodies against rubella a nd va ricella in infants. Vaccine 2011;29:2222-6.

[26] Goeyva erts N, Le uridan E, Fa es C, Va n Damme P, Hens N. Multi-disease analysis of maternal a ntibody decay using non linear mixed models accounting for censoring. Stat Med 2015;34:2858-71.

[27] Mell inger AK, Cragan JD, Atkinson WL, Williams WW, Kl eger B, Kimber RG, Ta vris D. High incidence of congenital rubella syndrome after a rubella outbreak. Pediatr Infect Dis J 1995;14:573-8.

[28] Pl otkin SA. Complex correlates of protection after va ccination. Clinical Infectious Diseases 2013;56:1458-65.

[29] Tischer A, Gassner M, Richard JL, Suter-Riniker F, Mankertz A, Heininger U. Va ccinated students with negative en zym e immunoassay results show positive measles vi rus-specific antibody l evels by i mm uno fluo rescence and plaque ne utralisation tests. J Cl in Virol 2007;38:204-9.

[30] Goeyva erts N, Hens N, The eten H, Aerts M, Van Damme P, Beutels P. Es timating va ccination cove rage for the trivalent mea sles-mumps-rubella va ccine from trivariate serological data. Stat in Med 2012;31:1432-49. 